\title{
Similarities and differences concerning flow characteristics in centrifugal compressors of different size
}

Elias Sundström, Bernhard Semlitsch and Mihai Mihăescu

\section{Introduction}

Downsizing of an automotive internal combustion engine is playing an important role for improving the energetic efficiency and reducing pollutant emissions [1]. Turbocharging is a key and matured technology, used for achieving these aims. However, its application range is bound to certain limits by appearance of unwanted fluid-flow phenomena leading to conditions like surge at low mass-flow rates and choke at high mass-flow rates [2]. The lack of detailed knowledge concerning the compressible flow behavior associated with turbo-machinery at off-design operating conditions imposes restrictive security margins on turbocharger's operating range. This is in order to avoid the occurrence of the violent flow phenomena, as e.g. surge, which can damage the compressor. Nevertheless, the surge line (indicating when surge occurs) is experienced to be individual for each compressor design and a generalization of surge limit to other designs is a desired but challenging task. Commonly, the surge phenomenon limits the application range more at the high rotational speed lines than at low rotational speed lines.

Gas stand measurements are carried out for experimental assessment of turbochargers, to quantify their performance parameters [3]. Such tests cannot give an indication about the three-dimensional flow and pressure fields associated with the different operating conditions. Nevertheless, the examination of the flow field is

Elias Sundström

Linné FLOW Centre and CCGEx, Department of Mechanics, KTH Royal Institute of Technology, Osquars Backe 18, 10044 Stockholm, Sweden, e-mail: elias@mech.kth.se

Bernhard Semlitsch

Linné FLOW Centre and CCGEx, Department of Mechanics, KTH Royal Institute of Technology, Osquars Backe 18, 10044 Stockholm, Sweden, e-mail: bernhard@mech.kth.se

Mihai Mihăescu

Linné FLOW Centre and CCGEx, Department of Mechanics, KTH Royal Institute of Technology, Osquars Backe 18, 10044 Stockholm, Sweden, e-mail: mihai@mech.kth.se 
relevant for understanding the behavior of the centrifugal compressor under various operating conditions.

In the present analysis, steady-state, three-dimensional Reynolds Averaged NavierStokes (RANS) simulations are used to explain the differences in the compressor performance maps for two automotive centrifugal compressors of different size. Special emphasis is put on the conditions closer to the surge line, over different speed lines.

\section{Geometry and Numerical Method}

The two investigated compressor geometries and the corresponding specifications are shown in Fig. 1. One is named small compressor (diffuser area ratio $=0.56$ ) and the other is named large compressor (diffuser area ratio $=0.62$ ). The surge bypass channel with a lock valve, the ball bearing features, and the impeller nut have been removed for simplification of the simulation setup. The inlet and volute exit pipes are extruded several diameters in the boundary normal directions, where the piping systems are equal for both compressors. Hence, an idealized installation of the compressor is considered (replicating the gas stand experiment) under so-called cold conditions with adiabatic wall boundary conditions.

In order to characterize the flow behavior occurring in the two compressors, a large number of operating conditions need to be simulated (different rotational speeds and mass-flow rates). Computational techniques based on the steady-state RANS formulation are relatively fast, robust, and affordable as compared with unsteady approaches [4], which can capture the mean flow features and trends corresponding to the measured global performance parameters. Despite their limitations, steady-state RANS modeling can be utilized for covering an impressive number of

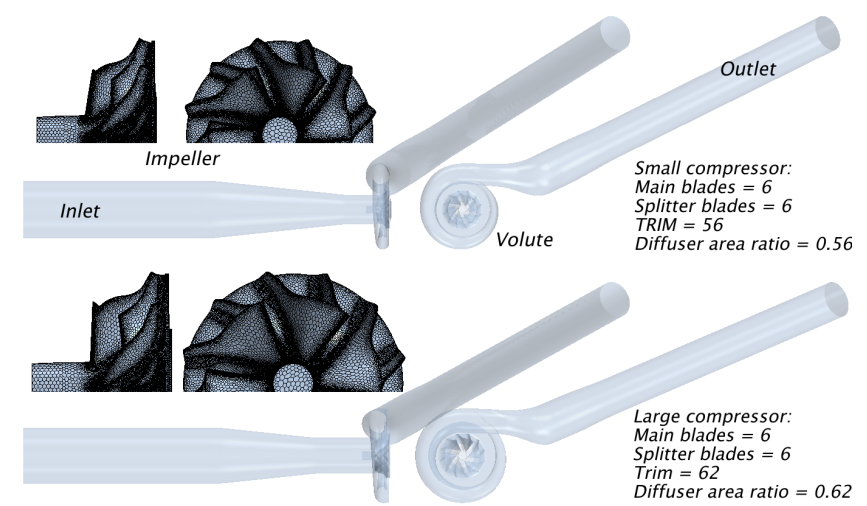

Fig. 1 Side and front views of the compressor geometry, small compressor at the top and the large compressor at the bottom. The mesh resolution is indicated in the enlarged impeller surface insets. 
cases within the stable compressor operating conditions, in a relatively short computational time [5].

Industry-standard modeling is employed to provide fast ( 3 hour) turn-around at each compressor operating point on a 6-core CPU workstation. The flow governing equations are solved by a compressible implicit coupled solver [6] based on the finite volume approach. The two-equation $k-\omega$ SST turbulence model is applied. About one million polyhedral computational cells are used to discretize the computational domain of each of the compressors, where two prism-layers are placed towards the no-slip walls. The Moving Reference Frame (MRF) approach is considered to take the effects of impeller's rotation into account. Mixing-plane interfaces are used which gives a circumferential averaged flow at the interfaces between the impeller region and the stationary. This treatment allows for mean radial variations.

\section{Results}

The global performance prediction in terms of the total-to-total pressure ratio and total-to-total efficiency are compared in Fig. 2 for the two compressor geometries. An overall good agreement between the numerical predictions and the experimental results could be achieved. A more pronounced humpback feature at the peak pressure ratios for the higher speed-lines is estimated by the computations. The geometrical simplifications for the numerical setup, as e.g. the removal of the surge by-pass channel, could potentially allow that higher pressure ratios are achieved at the higher speed-lines. The predicted total-to-total efficiency is in an overall good agreement
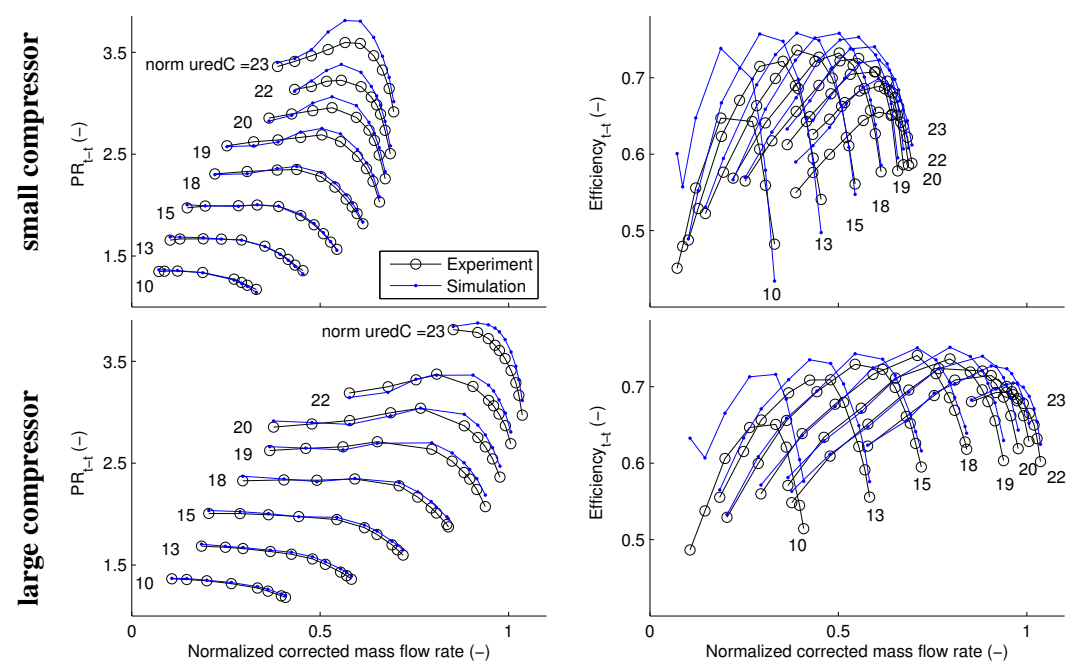

Fig. 2 Compressor pressure ratio (PR) and efficiency vs. normalized corrected mass flow. 
with the experimental data although the values are generally over-predicted by the simulation, where an improvement can be seen with higher mass flow rates. The over-prediction in absolute levels can be related to the not identical boundary conditions between the computational and experimental setup. Adiabatic wall treatment is employed in the simulation setup, whereas the turbocharger is running under socalled hot conditions in the experiment. Therefore the omission of wall heat transfer is seen to give an over-prediction at low mass flow rates, but at higher mass flow rates the assumption of an adiabatic wall becomes less crucial [7].

The flow field characteristics of the two investigated centrifugal compressors are given in Fig. 3 for the same impeller speed-line (normalized $u_{\text {redC }}=19$ ), which have been designed to operate in different mass-flow rate regimes. The smaller compressor is aimed for the usage at lower mass-flow rates, while the larger compressor can cope with larger mass-flow rates for e.g. a higher performance engine. The flow enters from the left into the domain and is accelerated towards the impeller. The compressor wheel is turning into the clockwise direction. At the tips of the wheel, the highest flow velocities are provoked. The imposed kinetic energy by the wheel blades is converted into pressure energy in the diffuser (the flow velocities decrease). From the diffuser, the stream exits tangentially into the volute. Since the volute increases its cross-sectional volume in clockwise direction (consistent with the wheel rotation), the pressure increases towards the outlet, see Fig. 3 (b). The observed flow features for the two compressor designs are similar at the near optimum efficiency operating condition.

Towards the surge line, lower mass-flow rates push against a high outlet pressure. The generated kinetic flow energy is not high enough to overcome the high static pressure built at the outlet and local flow reversal occurs. Thus, the flow is pushed from the diffuser region through the tip leakage (at the periphery of the impeller) against the main flow direction [8]. This is obvious for both compressors in Fig. 3 (a), where the annular high velocity flow can be seen at the outer periphery. This

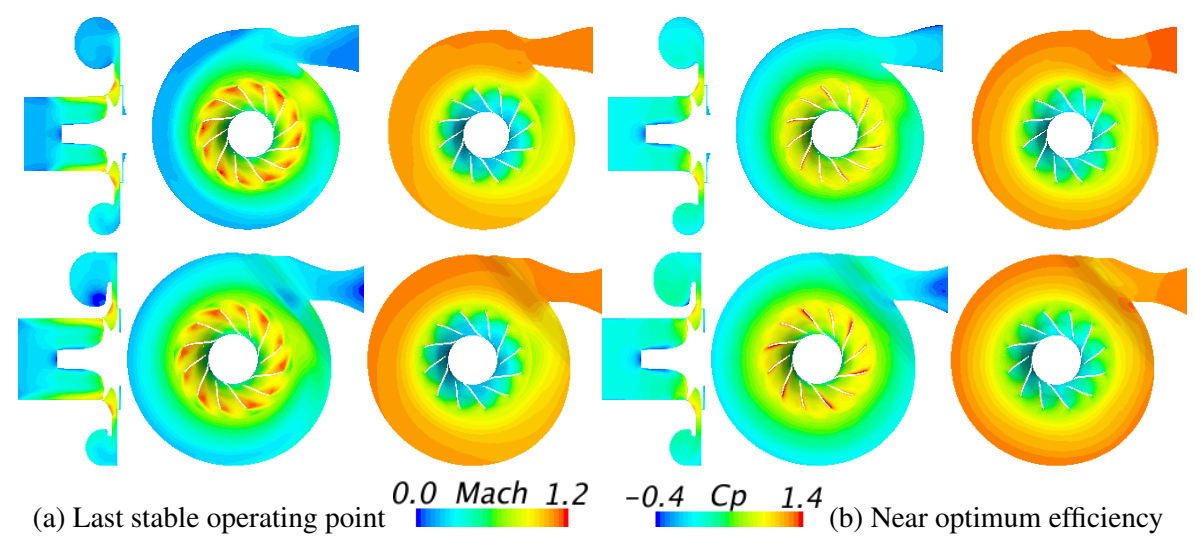

Fig. 3 Mach number and pressure coefficient contours in side and front view planes (small compressor at the top rows and large compressor at the bottom rows). 
flow feature is characteristic towards the surge margin and lowers the compressor performance. The reversed flow emerging from the tip leakage reaches far further upstream for the larger compressor. This is an effect of the larger compressor operating at higher mass-flow rates towards its surge line. The interaction between the reversed flow near the periphery of the impeller and the incoming flow triggers the surge occurrence at different locations in the compressor map.

At this near surge operating point, several differences in the flow field can be observed. The smaller compressor is capable to operate at lower mass flow rates, which results in lower flow velocities at the impeller inlet (see Fig. 3 (a)). This affects also the recirculating tip leakage flow, which is larger for the larger compressor. Another difference is the shift of the low-pressure region to the tongue region, which is slightly more pronounced for the small compressor. The compressor volute exhibits a significantly different design, which generates a different flow field appearance. For the large compressor, a design is chose allowing the flow to be attached at the outer volute walls. Generally, the same swirling motion as for the near optimum efficiency operating condition is induced in the volute, but a small stagnant flow region is provoked due to the low flow momentum. In contrary, the diffuser flow detaches from the walls and leaks centrally into the volute for the small compressor.

Figure 4 shows that the velocity upstream of the blades near the shroud increases with higher speed-lines for the last stable operating point, since the mass flow rate generally increases along the last stable point in the compressor map. The mass flow rate is lower near the surge-line for the lower speed-lines, which induces reduced velocities and the blades operate in an overall subsonic flow regime. In contrary, local sonic bubbles manifest at the blade tips for higher speed-lines, where the flow is accelerating over the suction side of the blades. Separated flow can be observed on the suction side of both the main and splitter blades. It commences on the mid-blade span of the main blades and extends to the rear of the blades. Towards the hub, the

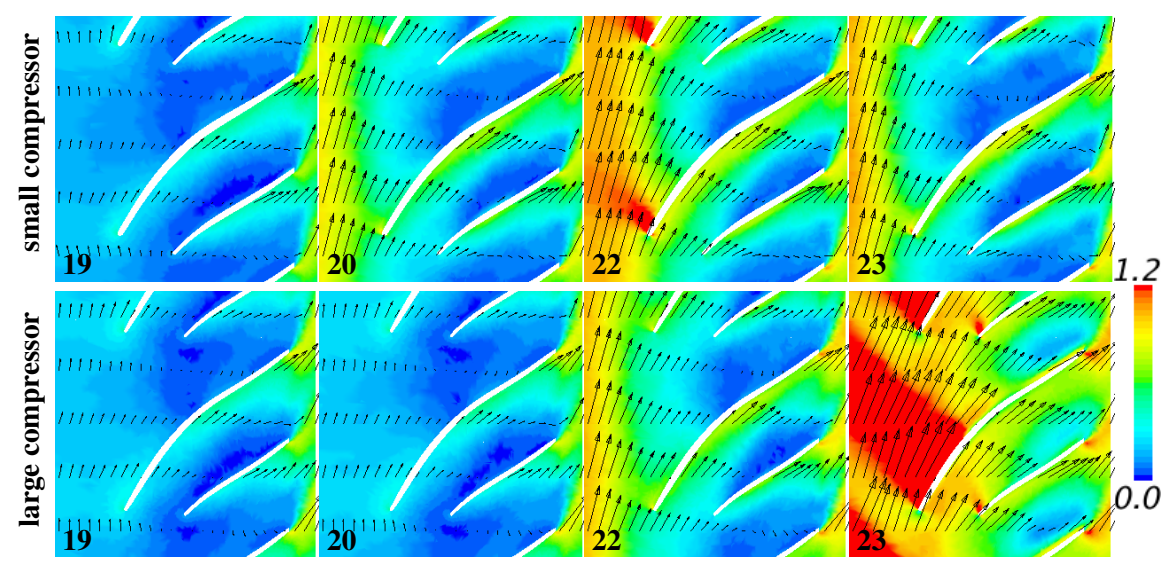

Fig. 4 Velocity vectors colored by the Mach number on a flattened surface at $85 \%$ blade span for the last stable operating point (increasing speed-lines $u_{\text {redC }}=19$ to $u_{\text {ured } C}=23$ from left to right). 
flow remains more attached at the blades, whereas close to the shroud occurs the tip leakage for these operating conditions. This flow separation decreases the impeller efficiency. By the diffuser inlet, the flow accelerates expanding in the volute.

\section{Conclusions}

The global compressor performance in terms of the pressure ratio and the efficiency are predicted numerically using a steady-state RANS approach for two different compressor geometries. A good agreement with measurements for an idealized installation are achieved for a vast range of operating conditions at different speedlines. The observed flow features for the two compressor designs are similar at the near optimum efficiency operating condition. However, towards the near surge operating condition, the different compressor design effects the flow field. The larger inducer diameter provokes lower velocities for a equal mass flow rates, which results in a less stable operating performance when approaching the surge line.

Acknowledgements The Competence Center for Gas Exchange (CCGEx), the Swedish Energy Agency (STEM), Volvo Group and SCANIA are acknowledged for supporting this project. The computations were performed on resources provided by the Swedish National Infrastructure for Computing (SNIC) at PDC Centre for High Performance Computing (PDC-HPC) and High Performance Computing Center North (HPC2N). Special thanks to BorgWarner for sharing the CAD geometries.

\section{References}

1. W. Knecht. Diesel engine development in view of reduced emission standards. Energy, 33(2), 264-271, 2008.

2. B. Semlitsch, V. JyothishKumar, M. Mihăescu, L. Fuchs, E. Gutmark. Investigation of the Surge Phenomena in a Centrifugal Compressor Using Large Eddy Simulation. ASME 2013 IMECE, IMECE2013-66301, 2013.

3. J. Galindo, F. Arnau, A. Tiseira, R. Lang, H. Lahjaily, T. Gimenes. Measurement and Modeling of Compressor Surge on Engine Test Bench for Different Intake Line Configurations. SAE Technical Paper, 2011-01-0370, 2013.

4. O. Baris, F. Mendonca. Automotive Turbocharger Compressor CFD and extension towards incorporating installation effects. ASME Turbo Expo, GT2011-46796, 2011.

5. E. Sundström, B. Semlitsch, M. Mihăescu. Assessment of the 3D flow in a centrifugal compressor using steady-state and unsteady flow solvers. SAE Technical Paper, 2014-01-2856, 2014.

6. STAR-CCM+, release version 10.02, CD-adapco, February 2015, www.cd-adapco.com.

7. J. Serrano, P. Olmeda, F. Arnau, M Reyes-Belmonte, A. Lefebvre. Importance of heat transfer phenomena in small turbochargers for passenger car applications. SAE Int. J. Eng. 6(2), 716728, 2013.

8. B. Semlitsch, V. JyothishKumar, M. Mihăescu, L. Fuchs, E. Gutmark, M. Gancedo. Numerical flow analysis of a centrifugal compressor with ported and without ported shroud. SAE Technical Paper, 2014-01-1655, 2014. 